\title{
Thoughts and Progress
}

\section{Chondrogenesis From Immortalized Human Mesenchymal Stem Cells: Comparison Between Collagen Gel and Pellet Culture Methods}

\author{
$*+C h i h-H u n g$ Chang, $¥ H \sin -Y i$ Lin, $\neq H s u$-Wei Fang, \\ †Siow-Tung Loo, \$Shih-Chieh Hung, *Yi-Ching Ho, \\ *Chia-Chun Chen, IIFeng-Huei Lin, \\ and II**Hwa-Chang Liu \\ *Department of Surgery, Division of Orthopedics, \\ Far Eastern Memorial Hospital, Taipei; + Graduate \\ School of Biotechnology and Bioengineering, Yuan \\ Ze University, Jung-Li, Tao Yuan; $\$$ Department of \\ Chemical Engineering and Biotechnology, National \\ Taipei University of Technology, Taipei; \$Institute of \\ Clinical Medicine, School of Medicine, National \\ Yang-Ming University, Taipei; IIInstitute of \\ Biomedical Engineering, National Taiwan \\ University, Taipei; and **Department of Orthopedic \\ Surgery, National Taiwan University Hospital, Taipei, \\ Taiwan
}

\begin{abstract}
Human mesenchymal stem cells (hMSCs) can differentiate into cells of connective tissue lineages, including cartilage. To overcome the limiting autogenous chondrocyte populations available in cartilage repair, various methods have been developed to maximize chondrogenesis of hMSCs in vitro, most of which use cells derived from primary culture. In this study, we compared chondrogenesis of immortalized hMSCs embedded in collagen gel to those grown in pellet culture. The hMSCs in collagen scaffolds expressed more glycosaminoglycan than those in pellet culture. Real-time reverse transcriptase-polymerase chain reaction (RT-PCR) analysis demonstrated that the expression of genes encoding sox-9, aggrecan, and types I and II collagen increased in pellet culture over time. However, in the collagen cultures, only type II collagen and aggrecan expression increased over time, whereas sox-9 expression remained unchanged and type I collagen expression decreased. These results indicate that the immortalized hMSC line is a promising tool for further in vitro chondrogenic studies. Key Words: Human mesenchymal stem
\end{abstract}

doi:10.1111/j.1525-1594.2008.00575.x

Received January 2007; revised September 2007

Address correspondence and reprint requests to Dr. Feng-Huei Lin, Institute of Biomedical Engineering, National Taiwan University, 7 Chung-Shan South Road, Taipei, 100, Taiwan. E-mail: double@ha.mc.ntu.edu.tw; or Dr Hwa-Chang Liu, Department of Orthopedic Surgery, National Taiwan University Hospital, Taipei, Taiwan.Email: hcliu@ntuh.gov.tw
cells-Cartilage tissue repair-Collagen scaffold-Biodegradable materials-Chondrogenesis.

Chondrocytes and mesenchymal stem cells (MSCs) are the predominant cell sources for cartilage tissue engineering. However, because of the limited proliferation ability of chondrocytes and their potential for loss of phenotype during monolayer expansion $(1,2)$, MSCs have become an attractive cell source.

Many methods have been used in chondrogenic induction of MSCs. Two commonly used methods are pellet culture and culturing in alginate beads or layers (3-6). The pellet culture method yields high cell density, which mimics the environment of embryonic cartilage development. However, this method results in a tightly aggregated cell mass with a large amount of cells forming only a small volume of tissue. Therefore, the number of cells needed to repair a large area of defective cartilage is considerable, and this problem is exacerbated by the fact that large pellets exhibit a periphery of viable cells, but the pellet center becomes necrotic (7). Also, pellets are difficult to handle and to mold into the various shapes needed for defect repair. These issues make pellet culture impractical for surgical applications.

Embedding human MSCs (hMSCs) in alginate or agarose gels $(8,9)$ has resulted in samples that could be cast into various shapes and that achieve substantial chondrogenesis. However, these hydrogels are not readily digestible by endogenous collagenase. A previous report (10) has shown that the gene encoding type II collagen was more strongly expressed in the alginate bead system than in the pellet culture system. In addition, the specific gene for hypertrophic cartilage, collagen type $\mathrm{X}$, was more rapidly expressed in the pellet system than in the alginate bead culture system. However, the drawbacks to using alginate scaffolds for cartilage tissue engineering include poor cell adhesion (11) and uncontrollable degradation of alginate following the diffusion of divalent cations into the surrounding medium (12). In addition, in vivo applications of alginate matrices have yielded severe foreign body giant cell reactions and immunological responses when implanted to treat full-thickness defects in cartilage in experimental animals $(13,14)$. Hence, alginate matrices have not been employed in human patients for articular cartilage repair. 
Collagen fibrils have a cell adhesion surface that transmits the biological information required for their function. Furthermore, the degradation products of collagen are physiological and nontoxic. Thus, type I collagen has generally been utilized as a carrier for chondrocytes or MSCs (15-17). The chondrogenesis of MSCs in collagen scaffolds has been examined in vivo, but in vitro studies have seldom been reported (18). In this study, we chose collagen to study MSC chondrogenic ability in vitro and compared the results to those obtained with the pellet culture system.

Most of the hMSCs used in previous cartilage tissue engineering studies were derived from primary cell cultures (3-5). Primary cells harvested from patients may have disease- or age-related differences such that results may be donor specific. We therefore chose to use an immortalized hMSC line to provide more consistent results for parametric studies designed to optimize tissue engineering procedures. The multi-lineage differentiation ability of this cell line has been reported (19), but chondrogenesis was only measured using Alcian blue staining and reverse transcription-polymerase chain reaction (RT-PCR) analysis of col2a1 expression. Therefore, in our current study, we compared hMSC chondrogenesis in pellet culture and collagen embedding systems via hematoxylin and eosin staining, immunohistochemistry, real-time RT-PCR, and analysis of glycosaminoglycan (GAG) content.

\section{MATERIALS AND METHODS}

\section{Cell culture and expansion of hMSCs}

The KP-hMSC line, an immortalized hMSC line, was cultured as described (19). Cells were cultured in Dulbecco's modified Eagle's medium with low glucose (DMEM-LG; GIBCO, Grand Island, NY, USA) containing $100 \mathrm{U} / \mathrm{mL}$ penicillin, $10 \mu \mathrm{g} / \mathrm{mL}$ streptomycin, $0.25 \mu \mathrm{g} / \mathrm{mL}$ amphotericin $\mathrm{B}$, and $10 \%$ fetal bovine serum (Biological Industries, Kibbutz Beit Haemek, Israel) at $37^{\circ} \mathrm{C}$ under $5 \%$ carbon dioxide and $95 \%$ humidity. The medium was changed twice a week. Cells were trypsinized and subcultured at a ratio of 1:3 every week.

\section{Pellet formation and chondrogenic induction}

KP-hMSC cultures were trypsinized, and $4 \times 10^{5}$ cells were centrifuged at $500 \times g$ for $10 \mathrm{~min}$. Cells were left in pellet form in the tubes overnight and became aggregated. The culture medium was replaced with chondrogenic medium, consisting of serum-free DMEM-LG containing ITS+ Premix $(6.25 \mu \mathrm{g} / \mathrm{mL}$ each of insulin, transferrin, and selenous acid; $1.25 \mathrm{mg} / \mathrm{mL}$ bovine serum albumin; and $5.35 \mu \mathrm{g} / \mathrm{mL}$ linoleic acid; Sigma, St. Louis, MO, USA), $50 \mu \mathrm{g} / \mathrm{mL}$ L-ascorbic acid-2-phosphate (Sigma), $0.1 \mu \mathrm{M}$ dexamethasone (Sigma), and $10 \mathrm{ng} / \mathrm{mL}$ TGF- $\beta 1$ (PeproTech, Rocky Hill, NJ, USA). The pellet medium was changed every 3 days, and cell pellets were cultured for 7,14, or 21 days with chondrogenic medium prior to analysis. Cells cultured in DMEM-LG containing only antibiotics and fetal bovine serum for 21 days were used as the pellet control. All pellets were cultured in $15 \mathrm{~mL}$ conical centrifuge tubes.

\section{Embedding hMSCs in an atelocollagen gel}

Trypsinized hMSCs were embedded in Porcogen collagen solution (3\% type I collagen, SunMax Biotech., Tainan County, Taiwan) containing chondrogenic medium. Eight volumes of Porcogen solution were added to 1 volume of DMEM-LG concentrated 10-fold, and 1 volume of $0.05 \mathrm{~N} \mathrm{NaOH}$, $2.2 \% \mathrm{NaHCO}_{3}$, and $200 \mathrm{mM}$ HEPES with gentle agitation at $0^{\circ} \mathrm{C}$. Cells were dispersed in the collagen medium mixture (final cell density $=2 \times 10^{6} \mathrm{cells} / \mathrm{mL}$ ), and $0.5 \mathrm{~mL}$ aliquots were allowed to gel in 24-well plates at $37^{\circ} \mathrm{C}$ for $1 \mathrm{~h}$ followed by the addition of $2.0 \mathrm{~mL}$ chondrogenic differentiation medium per well. The medium was changed every 3 days. The cell/collagen mixtures were analyzed 7,14 , and 21 days after cell seeding. Cell/collagen mixtures grown in DMEM-LG containing only antibiotics and fetal bovine serum for 21 days were used as the gel control.

\section{Histological analysis}

Both pellets and cell/collagen constructs were harvested after 7,14, or 21 days of culture and fixed in $10 \%$ formalin for $2 \mathrm{~h}$ at room temperature. The fixed pellets were dehydrated in increasing concentrations of ethanol, and cleared in xylene prior to embedding in paraffin. Sections of $5 \mu \mathrm{m}$ were cut, deparaffinized, and rehydrated by sequential immersion in 100\% xylene, $100 \%$ ethanol, $70 \%$ ethanol, and water. Hematoxylin and eosin staining (Muto, Tokyo, Japan) of sample sections was used to evaluate cell morphology in the pellets. Sulfated GAG was visualized by staining with Alcian blue (Sigma).

\section{Immunohistochemical analysis}

Cell pellet and cell/collagen constructs were incubated in $0.3 \% \mathrm{H}_{2} \mathrm{O}_{2}$ in methanol at room temperature for $15 \mathrm{~min}$ to block the activity of endogenous peroxidase. Nonspecific antibody binding was blocked by incubation of sections in $10 \%$ normal goat serum for $30 \mathrm{~min}$. Sections were then incubated with mouse anti-human type I collagen monoclonal antibody (1:100 dilution, Abcam Limited, Cambridge, UK) or polyclonal rabbit anti-human type II 
collagen (1:30 dilution, Novocastra Laboratories Ltd., Newcastle upon Tyne, UK) at room temperature for $1 \mathrm{~h}$. Staining was then visualized using the nonbiotin technique (Non-Biotin Detection kit HRP/DAB, BioGenex, San Ramon, CA, USA).

\section{Total GAG and DNA measurement}

Cell pellet and cell/collagen samples from days 7 , 14 , and 21 were washed with phosphate-buffered saline and digested with $0.5 \mathrm{~mL}$ of digest buffer $(1 \mu \mathrm{g} / \mathrm{mL}$ papain, $55 \mathrm{mM}$ sodium citrate, $150 \mathrm{mM}$ sodium chloride, $5 \mathrm{mM}$ cysteine hydrochloride, $5 \mathrm{mM}$ ethylenediaminetetraacetic acid [EDTA], $\mathrm{pH}$ 7.5) at $60^{\circ} \mathrm{C}$ for $16 \mathrm{~h}$ (all reagents were purchased from Sigma). Chondroitin 4-sulfate purified from bovine trachea (Blyscan Assay, Biocolor Ltd., Belfast, Northern Ireland) was used as a control. Standards and samples $(40 \mu \mathrm{L})$ were added to wells of a 96-well plate; $250 \mu \mathrm{L}$ of 1,9 dimethylmethylene blue (DMMB) solution was added to each well; and absorbance at $595 \mathrm{~nm}$ was determined using a microplate reader (VersaMax, Molecular Devices, Sunnyvale, CA, USA).

For DNA quantification, a standard curve was generated using double-stranded calf thymus DNA dissolved in PBE (100 $\mathrm{mM} \mathrm{Na}_{2} \mathrm{HPO}_{4}, 5 \mathrm{mM}$ EDTA, pH 7.5). Papain-digested samples were diluted with PBE. Standards and samples $(40 \mu \mathrm{L})$ were added in duplicate to wells of a 96-well plate to which $200 \mu \mathrm{L}$ of $0.2 \mu \mathrm{g} / \mathrm{mL}$ Hoechst 33258 dye (Sigma, St. Louise, Missouri, USA) in TN buffer (50 mM Tris, $150 \mathrm{mM} \mathrm{NaCl}$, $\mathrm{pH}$ 7.5) had been added. Excitation $(360 \mathrm{~nm})$ and emission $(460 \mathrm{~nm})$ for each sample were monitored using a Gemini II fluorescence plate reader (Molecular Devices). Analysis of variance was used for statistical analysis.

\section{RNA isolation and RT-PCR analysis of gene expression}

Total RNA was isolated using the RNeasy purification system according to the manufacturer's instructions (Qiagen, Valencia, CA, USA). Total RNA $(1 \mu \mathrm{g})$ was reverse transcribed using Superscript II RT (Invitrogen, Carlsbad, CA, USA). Gene expression was analyzed by real-time RT-PCR using an ABI Prism 7900HT sequence detection system (Applied Biosystems, Foster City, CA, USA). Primers for detection of human glyceraldehyde-3-phosphate dehydrogenase (endogenous control), aggrecan, sox-9, and collagen types I and II cDNAs were purchased from Applied Biosystems. A cycle threshold $(\mathrm{Ct})$ value was obtained for each sample, and triplicate sample values were averaged. The $2^{-\Delta \Delta \mathrm{Ct}}$ method was used to calculate relative expression of each target gene.

\section{RESULTS}

The engineered tissue constructs in collagen gels were generally larger than those in pellets. Histological analysis showed, however, that cells in collagen gels were less densely packed than in pellets (Fig. 1); Alcian blue staining (Fig. 1A-C) indicated an increase in sulfated GAG production from days 7 to 21 in cell/collagen culture. The increase of sulfated GAG production from days 7 to 21 in cell pellet culture was also observed by comparing the Alcian blue staining in Fig. 1D-F. Chondrocyte-like appearance with lacuna formation was occasionally observed in cell/collagen constructs (Fig. 1B, arrows), but not in the pellet or control groups. Immunohistochemical staining for type II collagen in both culture types is shown in Fig. 1G,H. DMMB staining to determine total GAG content is shown in Fig. 2. Cells in collagen gel culture produced significantly more $(P<0.05)$ GAG than cells in the pellet culture.

Temporal expression of genes involved in chondrogenesis was analyzed using real-time RT-PCR, as shown in Fig. 3. In the pellet culture (Fig. 3A), expression of type I collagen, type II collagen, aggrecan, and sox-9 increased over the 21-day test period relative to the pellet controls which lacked chondrogenic stimuli in the growth medium. In the cell/collagen construct (Fig. 3B), only type II collagen and aggrecan expression increased over the 21-day period. Type I collagen expression in the cell/collagen constructs initially increased eightfold by day 7 , but gradually decreased to approximately fivefold by day 21 . The expression of the sox-9 gene decreased the first 7 days and remained lower than the control throughout the experiment.

\section{DISCUSSION}

A previous study using Alcian blue staining of GAGs and real-time RT-PCR analysis of COL 2a1 expression suggested that the immortalized KPhMSC line was capable of chondrogenic differentiation (20). Here, we have expanded the real-time RT-PCR analysis to include other chondrogenic marker genes, and compared their expression and GAG content in cell pellet and collagen embedding systems commonly used for cartilage tissue engineering. The data show that GAG content increased over a 21-day period (Figs. 1 and 2). Likewise, real-time RT-PCR analysis revealed that expression of the aggrecan and type II collagen genes increased over the same period (Fig. 3). This is in agreement with a study by Yoneno et al. (18), in which GAG content was examined and real-time PCR was used to determine mRNA levels of collagen types II and X after 

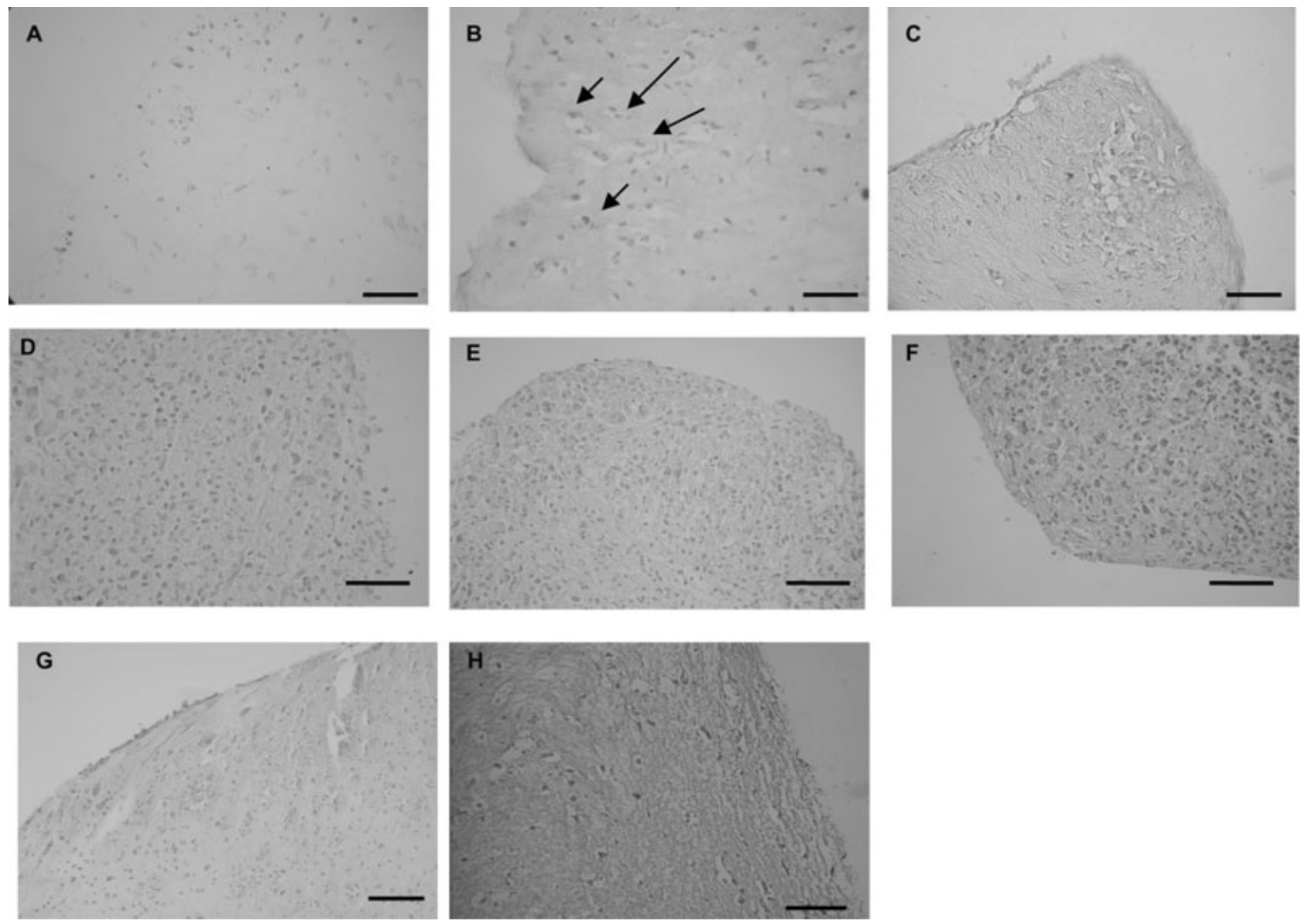

FIG. 1. Alcian blue staining and immunohistochemistry of hMSCs grown in collagen gel and pellet culture systems. Staining of sulfated GAGs from collagen gel $(A-C)$ and pellet $(D-F)$ culture systems grown in chondrogenic medium for $7(A, D), 14(B, E)$, and 21 (C, F) days. Cells were counterstained with eosin $Y$ in red. Scale bars in (A-C) are $100 \mu \mathrm{m}$; scale bars in (D-F) are $50 \mu \mathrm{m}$. Immunohistochemical staining of type II collagen from collagen gel $(G)$ and pellet $(H)$ culture systems grown in chondrogenic medium for 21 days is indicated by a reddish brown color. Scale bars in panels $\mathrm{G}$ and $\mathrm{H}$ are 100 and $50 \mu \mathrm{m}$, respectively.

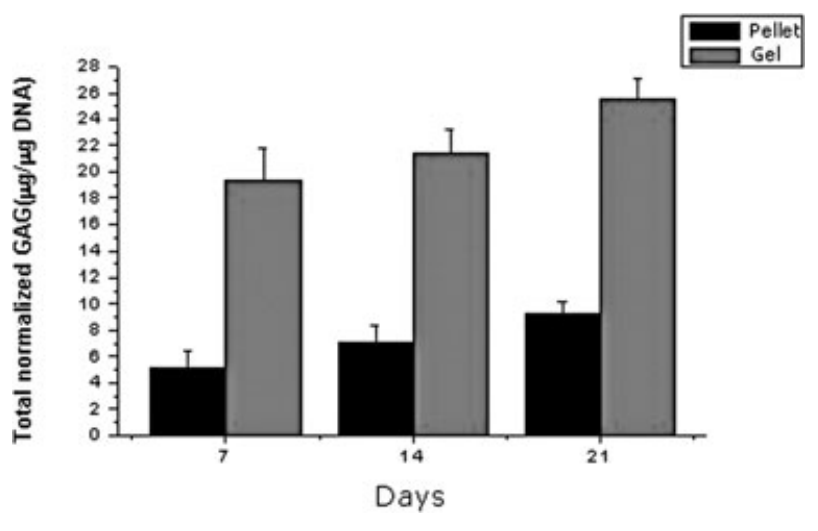

FIG. 2. Comparison of total GAG content from collagen gel culture and pellet culture systems. Total GAG content in the cell/collagen constructs (black) and pellets (gray) was normalized to total DNA content. Data shown are an average of triplicate $(n=3)$ samples. Data are averages ( \pm standard deviation) of three experiments in which triplicate samples were assessed. chondrogenic stimulation of collagen-embedded hMSCs believed to be from primary cell culture. Although type X collagen was not tested in our study, Yoneno et al. demonstrated that type $\mathrm{X}$ collagen mRNA increased until day 15 and decreased thereafter. Barry et al. (20) analyzed chondrogenic differentiation of primary hMSCs in pellet culture using RT-PCR, and determined that type I collagen mRNA was uniformly detected in the undifferentiated cells and throughout differentiation, but type II collagen mRNA was only detected after 8 days in pellet culture and gradually increased thereafter. In addition, AGN expression reached consistent levels within 4-5 days in the pellet culture system of Barry et al. These discrepancies between our results and previous literatures likely reflect differences between primary and immortalized cell cultures, and/or differences between techniques used to detect transcript levels. 

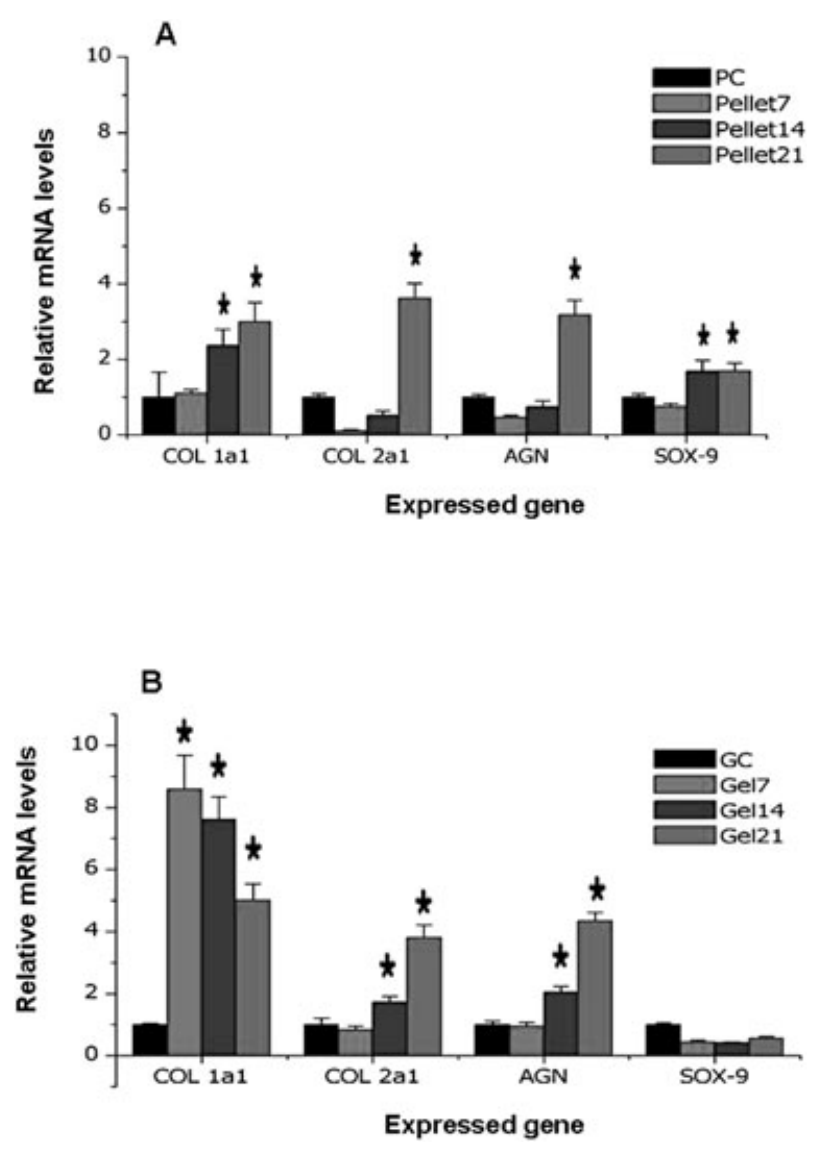

FIG. 3. Real-time RT-PCR analysis to compare gene expression profiles from collagen gel culture and pellet culture systems. RT-PCR analysis of various gene transcripts from hMSCs cultured in pellets $(A)$ or in collagen gels (B). Pellet7, Pellet14, and Pellet21 represent pellet samples cultured in chondrogenic medium for 7,14 , or 21 days, respectively. PC is pellet control lacking chondrogenic stimulation. Gel7, Gel14, and Gel21 represent collagen gel samples cultured in chondrogenic medium for 7,14 , or 21 days, respectively. GC is collagen gel control lacking chondrogenic stimulation. COL 1a1, type I collagen; COL 2a1, type II collagen; AGN, aggrecan. ( ${ }^{\star} P<0.05$.)

Importantly, we have demonstrated that chondrogenesis of hMSC can be induced in the collagen gel system, which is a commonly used scaffold material for cartilage tissue repair. The collagen culture was superior to the pellet culture because cells in the collagen gel had more GAG expression per unit DNA (Fig. 2) and more lacuna formation (Fig. 1B). In addition, the initially high expression of type I collagen gene decreased over time, whereas type II collagen gene expression in the collagen matrix increased gradually over time (Fig. 3B). Excess type I collagen production in repaired cartilage induces fibrocartilage formation, suggesting that the collagen gel system is favorable for hyaline cartilage formation. The amount of type I collagen secretion depends on the rate at which the MSCs differentiate. A variety of hormones, growth factors, and local factors may be able to regulate type I collagen secretion (21). The type I collagen gel used in this study was made from porcine epithelial tissues according to the manufacturer's specification. It might contain trace amounts of growth factors or hormones that induced MSC to express type I collagen and result in the initial increase of type I collagen mRNA expression. As the amount of trace hormones and growth factors dissolved into media over time and were removed through media replacement, type I collagen gene expression decreased as well. Bosnakovski et al. (22) observed similar results. They contributed the result to the interaction between $\alpha 2$ integrin subunit on type I collagen and the collagen type I receptor on MSCs. The interaction leads to MSCs' osteoblastic differentiation, and thus, the increase of mRNA of type I collagen.

sox-9 expression is important for chondrogenic differentiation after mesenchymal condensation, and inhibits the transition from chondrocyte proliferation to hypertrophy (23), a process that is detrimental to tissue engineering because it can lead to apoptosis, vascular invasion, and calcification. Our analysis indicated that sox-9 expression remained low in the collagen embedding system, but increased in the pellet culture system. Although Yoneno et al.'s study (18) showed decreased type $\mathrm{X}$ collagen expression after a 15-day culture period and no chondrocyte hypertrophy using the collagen embedding system, the low expression of sox-9 measured in our present study raises the concern that the collagen embedding system may compromise the inhibition of chondrocyte hypertrophy. Currently, methods to inhibit chondrocyte hypertrophy from MSC chondrogenesis in pellet culture are imperfect $(4,24)$, and thus further study is necessary to clarify the hypertrophy of MSC chondrogenesis using the collagen embedding method.

\section{CONCLUSION}

Our study shows that, like MSCs from primary culture, immortalized hMSCs can be used for chondrogenic studies in both pellet and collagen embedding systems, and are therefore a promising tool for further chondrogenic studies. Our results indicate that the collagen culture system seems to be superior to pellet culture, but the data also raise concerns about the hypertrophy of MSC chondrogenesis in this method.

Acknowledgments: We thank the National Science Council of Taiwan (NSC-96-2627-B-002-001) and Far Eastern Memorial Hospital (FEMH-95-C-006) for financial support. 


\section{REFERENCES}

1. Goldring MB, Sandell LJ, Stephenson ML, Krane SM. Immune interferon suppresses levels of procollagen mRNA and type II collagen synthesis in cultured human articular and costal chondrocytes. J Biol Chem 1986;261:9049-55.

2. Saadeh PB, Brent B, Mehrara BJ, Steinbrech DS, Ting V, Gittes GK, Longaker MT. Human cartilage engineering: chondrocyte extraction, proliferation, and characterization for construct development. Ann Plast Surg 1999;42:509-13.

3. Johnstone B, Hering TM, Caplan AI, Goldberg VM, Yoo JU. In vitro chondrogenesis of bone marrow derived mesenchymal progenitor cells. Exp Cell Res 1998;238:265-72.

4. Mackay AM, Beck SC, Murphy JM, Barry FP, Chichester CO, Pittenger MF. Chondrogenic differentiation of cultured human mesenchymal stem cells from marrow. Tissue Eng 1998;4:415-28.

5. Hung SC, Chen NJ, Hsieh SL, Li H, Ma HL, Lo WH. Isolation and characterization of size-sieved stem cells from human bone marrow. Stem Cells 2002;20:249-58.

6. Woodward WA, Tuan RS. $N$-Cadherin expression and signaling in limb mesenchymal chondrogenesis: stimulation by polyL-lysine. Dev Genet 1999;24:178-87.

7. Kavalkovich KW, Boynton RE, Murphy JM, Barry F. Chondrogenic differentiation of human mesenchymal stem cells within an alginate layer culture system. In Vitro Cell Dev Biol Anim 2002;38:457-66.

8. Huang CY, Reuben PM, D'Ippolito G, Schiller PC, Cheung HS. Chondrogenesis of human bone marrow-derived mesenchymal stem cells in agarose culture. Anat Rec A Discov Mol Cell Evol Biol 2004;278:428-36.

9. Ma HL, Hung SC, Lin SY, Chen YL, Lo WH. Chondrogenesis of human mesenchymal stem cells encapsulated in alginate beads. J Biomed Mater Res A 2003;64:273-81.

10. Yang IH, Kim SH, Kim YH, Sun HJ, Kim SJ, Lee JW. Comparison of phenotypic characterization between "alginate bead" and "pellet" culture systems as chondrogenic differentiation models for human mesenchymal stem cells. Yonsei Med $J$ 2004;45:891-900.

11. Chung TW, Yang J, Akaike T, Cho KY, Nah JW, Kim SI, Cho CS. Preparation of alginate/galactosylated chitosan scaffold for hepatocyte attachment. Biomaterials 2002;23:2827-34.

12. Atala A, Lanza RP (eds). Methods in Tissue Engineering, 1st Edition. Elsevier, NY: Academic Press, 2002.

13. Hunziker EB. Articular cartilage repair: basic science and clinical progress. A review of the current status and prospects. Osteoarthritis Cartilage 2001;10:432-63.

14. Perka C, Schultz O, Spitzer R, Lindenhayn K. The influence of transforming growth factor beta1 on mesenchymal cell repair of full-thickness cartilage defects. J Biomed Mater Res 2000; 52:543-52.

15. Kawamura S, Wakitani S, Kimura T, Maeda A, Caplan AI, Shino K. Articular cartilage repair: rabbit experiments with a collagen gel-biomatrix and chondrocytes cultured in it. Acta Orthop Scand 1998;69:56-62.

16. Frenkel S, Toolan B, Menche D, Pitman M, Pachence J. Chondrocyte transplantation using a collagen bilayer matrix for cartilage repair. J Bone Joint Surg 1997;79B:831-6.

17. Im G, Kim D, Shin J, Hyun C, Cho W. Repair of cartilage defect in the rabbit with cultured mesenchymal stem cells from bone-marrow. J Bone Joint Surg 2001;83B:289-94.

18. Yoneno K, Ohno S, Tanimoto K, Honda K, Tanaka N, Doi T, Kawata T, Tanaka E, Kapila S, Tanne K. Multidifferentiation potential of mesenchymal stem cells in three-dimensional collagen gel cultures. J Biomed Mater Res A 2005;75:733-41.

19. Hung SC, Yang DM, Chang CF, Lin RJ, Wang JS, Low-Tone Ho L, Yang WK. Immortalization without neoplastic transformation of human mesenchymal stem cells by transduction with HPV16 E6/E7 genes. Int J Cancer 2004;110:313-19.

20. Barry F, Boynton RE, Liu B, Murphy M. Chondrogenic differentiation of mesenchymal stem cells from bone marrow: differentiation-dependent gene expression of matrix components. Exp Cell Res 2001;268:189-200.

21. Bord S, Frith E, Ireland DC, Scott MA, Craig JI, Compston JE. Megakaryocytes modulate osteoblast synthesis of type- 1 collagen, osteoprotegerin, and RANKL. Bone 2005;36:812-9.

22. Bosnakovski D, Mizuno M, Kim G, Takagi S, Okumura M, Fujinaga T. Chondrogenic differentiation of bovine bone marrow mesenchymal stem cells (MSCs) in different hydrogels: influence of collagen type II extracellular matrix on MSC chondrogenesis. Biotechnol Bioeng 2006;93:1152-63.

23. Ikeda T, Kawaguchi H, Kamekura S, Ogata N, Mori Y, Nakamura K, Ikegawa S, Chung UI. Distinct roles of Sox5, Sox6, and Sox 9 in different stages of chondrogenic differentiation. J Bone Miner Metab 2005;23:337-40.

24. Sekiya I, Vuoristo JT, Larson BL, Prockop DJ. In vitro cartilage formation by human adult stem cells from bone marrow stroma defines the sequence of cellular and molecular events during chondrogenesis. Proc Natl Acad Sci USA 2002;99:4397-402.

\section{Laboratory Performance Testing of Venous Cannulae During Inlet Obstruction}

\author{
*Antoine P. Simons, †Yuri Ganushchak, \\ †Pieter Wortel, *Theo van der Nagel, \\ *Frederik H. van der Veen, †Dick S. de Jong, \\ and *Jos G. Maessen \\ *Department of Cardiothoracic Surgery, \\ Cardiovascular Research Institute Maastricht, \\ University Hospital Maastricht; and †Department of \\ Extra-Corporeal Circulation, Cardiovascular \\ Research Institute Maastricht, University Hospital \\ Maastricht, Maastricht, The Netherlands
}

Abstract: Venous cannulae undergo continuous improvements to achieve better and safer venous drainage. Several cannula tests have been reported, though cannula performance during inlet obstruction has never been a test criterion. In this study, five different cannulae for proximal venous drainage were tested in a mock circulation that enabled measurement of hydraulic conductance after inlet obstruction by vessel collapse. Values for hydraulic conductance ranged from $1.11 \times 10^{-2} \mathrm{~L} / \mathrm{min} /$ $\mathrm{mm} \mathrm{Hg}$ for a Thin-Flex Single Stage Venous Cannula with an open-end lighthouse tip to $1.55 \times 10^{-2} \mathrm{~L} / \mathrm{min} / \mathrm{mm} \mathrm{Hg}$ for a DLP VAD Venous Cannula featuring a swirled tip profile, showing a difference that amounts to nearly $40 \%$ of the lowest conductance value. Excessive venous drainage results in potentially dangerous high-negative venous line pressures independent of cannula design. Cannula tip design featuring swirled and grooved tip structures

doi:10.1111/j.1525-1594.2008.00584.x

Presented in part at the 12th European Congress on Extracorporeal Circulation Technology held June 6-9, 2007 in Kiev, Ukraine.

Received July 2007; revised September 2007.

Address correspondence and reprint requests to: Antoine P. Simons, MSc, University Hospital Maastricht, Department of Cardiothoracic Surgery (CTC), P. Debyelaan 25, P.O. Box 5800, 6202 AZ Maastricht, The Netherlands. E-mail: antoinepsimons@ctc. unimaas.nl 